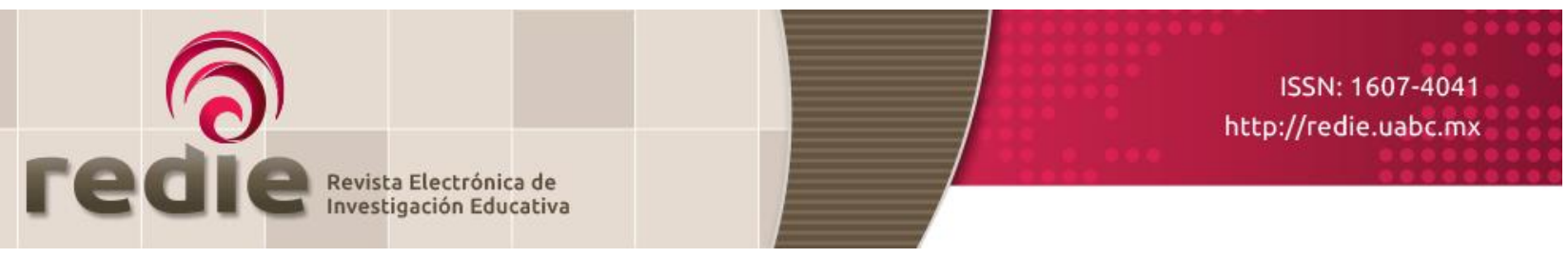

Vol. 19, Núm. 1, 2017

\title{
Evidencias de ambigüedad de rol en profesores universitarios
}

\section{Indications of Role Ambiguity in University Teachers}

\author{
Edith Georgina Surdez Pérez (*) edith.2109@hotmail.com \\ Deneb Elí Magaña Medina (*) deneb 72@yahoo.com \\ María del Carmen Sandoval Caraveo (*) sandovalcaraveo29@hotmail.com
}

(*) Universidad Juárez Autónoma de Tabasco

(Recibido: 17 de septiembre de 2014; Aceptado para su publicación: 12 de octubre de 2015)

Cómo citar: Surdez, E. G., Magaña, D. E. y Sandoval, M. del C. (2017). Evidencias de ambigüedad de rol en profesores universitarios. Revista Electrónica de Investigación Educativa, 19(1), 73-83. Recuperado de https://doi.org/10.24320/redie.2017.19.1.889

\section{Resumen}

La ambigüedad de rol afecta negativamente la satisfacción laboral y los logros organizacionales en el ámbito educativo. El objetivo de esta investigación fue determinar evidencias de ambigüedad de rol en profesores universitarios que comparten su tiempo entre actividades de docencia y de investigación en el área de Ciencias Sociales y Administrativas. El estudio se realizó en una institución de educación superior pública en México, es descriptivo y correlacional, con enfoque cuantitativo. Se utilizó una escala tipo Likert, con una confiabilidad de .92 de coeficiente alfa de Cronbach y cargas factoriales arriba de .60 en todas las dimensiones. Los resultados reportan que los niveles más altos se presentan en las dimensiones: Ambigüedad en las Demandas y Ambigüedad en las Normas, principalmente por la falta de claridad en los indicadores y lineamientos que rigen las evaluaciones sobre el desempeño académico.

Palabras clave: Universidad, rol del profesor, evaluación del profesor.

\section{Abstract}

Role ambiguity has a negative effect on job satisfaction and organizational achievements in the education field. The aim of this research was to identify indications of role ambiguity in university teachers who split their time between teaching and research in the field of social and management sciences. The study, performed in a public higher education institution in Mexico, is descriptive and correlational and uses a quantitative approach. A Likert-type scale was used with a Cronbach's alpha reliability coefficient of .92 and factor loadings above .60 for all dimensions. The results find the highest levels in the dimensions "Ambiguity in Demands" and "Ambiguity in Standards", mainly caused by a lack of clarity in the guidelines and framework that regulate the assessment of academic performance. 


\section{Introducción}

La ambigüedad de rol se puede presentar en el profesor universitario debido a que legendariamente se ha considerado deseable que comparta su dedicación y tiempo entre la docencia y la investigación. Desde el siglo XIX, en la fundación de la Universidad de Berlín se estableció como principio perdurable la unidad entre investigación y docencia, así también, la vinculación enseñanza-investigación fue la marca en las universidades americanas desde ese tiempo, y en el siglo XX en las instituciones de educación superior latinoamericanas a partir de la Reforma Universitaria de Córdoba (Sebastián, 2003). Esta doble función, aunada a nuevas demandas para los profesores en el ámbito de la educación superior, agudiza la problemática de ambigüedad de rol.

Por una parte, los nuevos modelos educativos, tales como el de formación integral del estudiante, el basado en competencias, el proceso de armonización de materias de enseñanza superior, entre otros, que implican una ampliación cuantitativa de las actividades de enseñanza-aprendizaje que conlleva a desempeñar no sólo el rol tradicional de docente frente a un grupo, sino el de supervisor de prácticas y trabajo de campo (Troyano, García y Marín, 2006).

En cuanto al rol de investigador se pueden mencionar, como nuevas demandas, la transición de la investigación basada en la individualidad a la generada por grupos de investigación y la tendencia hacia formas organizativas más complejas en las actividades de investigación, como la realización de proyectos en los que participan diversos investigadores, instituciones y países a través de redes de investigación (Sebastián, 1999b, como se cita en Sebastián, 2003) o como en el caso de México a través de la conformación de grupos de investigación que reciben el nombre de Cuerpos Académicos. ${ }^{1}$

Por otra parte, los modelos de racionalidad organizacional bajo los que actualmente operan las universidades, en donde los recursos se asignan con base en la evaluación de resultados, mediante la cuantificación del desempeño académico. Esto ocasiona un comportamiento utilitario en algunos sujetos, produciéndose en ellos una preocupación y ocupación económica individual que desplaza su contribución a las funciones sustantivas de la universidad (Ibarra, 1999).

En este contexto, en México a partir de la década de los noventa, para hacer frente a los recursos económicos limitados, inflación y pérdida del poder adquisitivo de los salarios, circunstancias derivadas de la crisis a partir de los años ochenta, se establecieron sistemas de medición de la calidad académica y la productividad de los profesores universitarios (Soto, 1997; Izquierdo, Sandoval, Eslava y De la Cruz, 2013), a través de los cuales se otorgan incentivos económicos con fines compensatorios o complementarios al sueldo del profesor (Aquino, 2005), como el Programa para el Desarrollo Profesional Docente (PRODEP), ${ }^{2}$ el Estímulo al Desempeño del Personal Docente ${ }^{3}$ y el Sistema Nacional de Investigadores (SNI). ${ }^{4}$

Estos programas tienen enfoques y requerimientos diferentes, los dos primeros valoran el equilibrio entre docencia, investigación, tutoría y gestión académica y el último enfatiza la productividad generada de la investigación (Secretaría de Educación Pública, 2013; Universidad Juárez Autónoma de Tabasco,

\footnotetext{
1 "Grupos de profesores/as de tiempo completo que comparten una o varias líneas de generación de conocimiento, investigación aplicada o desarrollo tecnológico e innovación en temas disciplinares o multidisciplinares y un conjunto de objetivos y metas académicas" (Secretaría de Educación Pública, 2013)

${ }^{2}$ Programa que tiene como objetivo contribuir para que los Profesores de Tiempo Completo (PTC) de las instituciones públicas de educación superior alcancen las capacidades para realizar investigación-docencia, se profesionalicen, se articulen y se consoliden en cuerpos académicos (Secretaría de Educación Pública, 2013).

${ }^{3}$ Estímulos que la Unidad Política y Control Presupuestal de la Secretaría de Hacienda y Crédito Público, a través de la Secretaría de Educación Pública, otorga al desempeño del personal docente (Universidad Juárez Autónoma de Tabasco, 2014)

4 “El Sistema Nacional de Investigadores (SNI) fue creado por acuerdo presidencial publicado en el Diario Oficial de la Federación el 26 de julio de 1984, para reconocer la labor de las personas dedicadas a producir conocimiento científico y tecnología. El reconocimiento se otorga a través de la evaluación por pares y consiste en otorgar el nombramiento de investigador nacional. Esta distinción simboliza la calidad y prestigio de las contribuciones científicas. En paralelo al nombramiento se otorgan estímulos económicos cuyo monto varía con el nivel asignado" (Consejo Nacional de Ciencia y Tecnología, 2014).
} 
2014; Consejo Nacional de Ciencia y Tecnología, 2014). Ante esta realidad el profesor ha tenido que hacer frente a nuevas demandas de trabajo, en ocasiones sin estar suficientemente informado sobre lo que entrañan estas nuevas responsabilidades.

En este orden de ideas, es importante que las instituciones de educación superior identifiquen las condiciones institucionales que pueden generar ambigüedad de rol, debido a que este fenómeno obstaculiza el logro de objetivos organizacionales y genera malestar en el profesorado. En este documento se presentan los resultados de una investigación cuyo objetivo fue determinar evidencias de ambigüedad de rol en los profesores investigadores afiliados a cuerpos académicos del área de conocimiento de Ciencias Sociales y Administrativas de una institución de educación superior pública estatal en México; incluye la División Académica de Ciencias Económico, Administrativas, la División Académica de Ciencias Sociales y Humanidades y la División Académica de Educación y Artes.

\section{Conceptualización y etiología de la ambigüedad de rol}

En una organización, el "rol" consiste en el conjunto de patrones de conducta que se esperan del individuo que ocupa una posición en determinada unidad social (Robbins y Coulter, 1999). Para De Arquer, Daza y Nogareda (1995) la ambigüedad de rol es la falta de definición respecto al rol que debe desempeñar un individuo en una organización; por información incompleta, poco concisa o muy cambiante sobre objetivos de trabajo, responsabilidades, relaciones, autoridad y procedimientos; argumentan que la persona con ambigüedad de rol vive en la incertidumbre, no sabe qué se espera de ella, es decir, no tiene configurado con claridad cuál es su rol en la organización. Para Goldman y Chang (1992) la ambigüedad de rol es la discrepancia entre la información disponible para el individuo en una organización y la que requiere para el desempeño adecuado de sus funciones. A su vez, León-Rubio, Cantero y León-Pérez (2011) consideran la ambigüedad de rol como información inadecuada o poco clara de los objetivos asociados a un rol y sobre las expectativas de los otros respecto al rol, como desconocer los criterios a través de los cuales se le evaluará para un ascenso.

La ambigüedad de rol ha sido un constructo válido en investigaciones sobre comportamiento organizacional debido a que ha habido una evaluación suficiente (meta-análisis) que indica que el promedio de las correlaciones entre considerables variables de contexto organizacional, tales como autonomía, retroalimentación de otros, identidad de la tarea, estructura de la iniciativa del líder, entre otras, y la ambigüedad de rol son sustanciales (Jackson y Schuler, 1985).

Como causas de la ambigüedad de rol se consideran varios aspectos del contexto organizacional y características individuales (Jackson y Schuler, 1985). En cuanto a los factores organizacionales relacionados con propensión a la ambigüedad de rol, los mencionados en la literatura son: escasez de estandarización en el trabajo y de autonomía (Lloret, González y Peiró, 1995), falta de claridad sobre los objetivos del trabajo individual o el alcance de las responsabilidades (Slipak, 1996), desconocimiento de expectativas asociadas al rol (Malone, 2002), inadecuado ambiente de comunicación (Schulz y Auld, 2006), insuficiente retroalimentación de otros y de identidad de la tarea (Jackson y Schuler, 1985), con liderazgo y prácticas organizacionales variadas en un patrón de la teórica clásica (Rizzo, House y Lirtzman, 1970). Webb (1990, como se cita en Zepeda y Kruskamp, 2007) identifica seis factores que contribuyen a la ambigüedad de rol: equivocada descripción del trabajo, funciones contradictorias, metas vagas, desarrollo del personal ineficaz, falta de acuerdo entre el director y los administradores centrales y recursos inadecuados.

Ahora bien, de las características individuales relacionadas con la ambigüedad de rol se ha hallado que el locus de control y la antigüedad laboral (que genera experiencia) moderan las reacciones de los empleados a la ambigüedad. Por otra parte, las correlaciones de edad y escolaridad con ambigüedad de rol son siempre muy débiles (Jackson y Schuler, 1985; Schulz y Auld, 2006), es decir, en la percepción de ambigüedad de rol por parte de un trabajador no influye la preparación académica o el que se encuentre en etapas más avanzadas del ciclo de vida, lo que confirma que esta problemática está más asociada a circunstancias laborales. 


\subsection{Implicaciones de la ambigüedad de rol}

Diversos estudios reportan que la ambigüedad de rol tiene consecuencias negativas para la organización y para el individuo, y en varios de estos el sujeto de estudio es el profesor universitario. Schulz (2013) expone que el conflicto y la ambigüedad de rol son relativamente comunes en las universidades, ya que los estudiantes demandan a los profesores tiempo y atención y las plazas universitarias solicitan investigación y publicaciones. Gomley (2005) en un estudio que abarca docencia, investigación y servicio en profesores de diversas facultades de Enfermería concluye que la ambigüedad de rol propicia que los individuos se sientan menos acoplados a la organización, que experimenten obstáculos y poco goce en las relaciones con colegas, que el énfasis en la productividad disminuya, renuncias, estrés, tensión, baja satisfacción y baja motivación.

A su vez, Malone (2002), en un estudio sobre profesores universitarios de nuevo ingreso, encontró que la ambigüedad de rol fue el factor de estrés más reportado. Edmonson y Thompson (2001) en un metaanálisis para investigar el impacto de la ambigüedad y el conflicto de rol en el agotamiento de maestros de educación especial, identificaron que la ambigüedad de rol incrementa el desgaste emocional y la despersonalización. León-Rubio et al. (2011), al encuestar a 194 docentes investigadores de la Universidad de Sevilla, encontraron que a mayor ambigüedad de rol mayor proporción de personas con el atributo burnout. Gismero-González et al. (2012) reportaron en una muestra de 413 docentes de de Madrid, que el estrés causado por el conflicto y la ambigüedad de rol es afrontado con el uso de una estrategia de pasividad pesimista, que implica centrarse en los aspectos de la situación estresante, atribuyendo sus causas a factores externos y resignándose a ellos.

Asimismo, se han realizado estudios para medir el impacto de la ambigüedad de rol en la satisfacción laboral, al respecto Meliá, Zarnoza, Sanz, Morte y González (1987), en un estudio en el que los participantes fueron trabajadores, supervisores, mandos intermedios y directivos del sector empresarial, identificaron que factores de ambigüedad de rol relativos al puesto de trabajo como son la cantidad y claridad de la información sobre: los límites de las responsabilidades y competencias, los límites de la autoridad, las tareas del puesto de trabajo y los procedimientos para realizarlas, objetivos, nivel de producción y calidad a alcanzar, así como la claridad de las expectativas de superiores, compañeros y subordinados del trabajador y la ambigüedad sobre derechos laborales y sociales están relacionados significativa y negativamente con la satisfacción laboral.

A su vez, Schulz (2013), en un estudio en el que participaron académicos de universidades en donde se realiza investigación en forma intensa (Rusell Group Universities), encontró que la ambigüedad de rol tiene un efecto negativo en la satisfacción, y que el tipo de clima laboral influye en esta relación, es así que el clima laboral jerárquico se asocia a bajos niveles de ambigüedad. Sin embargo no tiene impacto en la satisfacción laboral, en contraposición el clima de tipo clan, que propicia un ambiente de apoyo, reduce la ambigüedad y contribuye positivamente a la satisfacción laboral.

\section{Método}

El estudio es descriptivo y correlacional, con enfoque cuantitativo, con diseño no experimental transversal. La población del área de conocimiento de Ciencias Sociales y Administrativas (División Académica de Ciencias Económico-Administrativas, División Académica de Ciencias Sociales y Humanidades y División Académica de Educación y Artes) la conforman 127 profesores-investigadores de 15 cuerpos académicos distribuidos en las tres divisiones. El muestreo empleado fue el Estratificado, tomando como estrato cada una de las divisiones académicas de una universidad pública estatal de México. La muestra de cada estrato se determinó de forma proporcional considerando el tamaño de la población de cada división académica, con un error de estimación del 5\% y una confiabilidad del 95\%. La selección de los profesores fue sistemática, esto significó para el área de Ciencias Sociales y Administrativas una participación de 64 elementos, como se observa en la tabla I. 
Tabla I. Muestreo estratificado de las divisiones académicas

\begin{tabular}{lcc}
\hline División Académica & Población & Muestra \\
\hline División Académica de Ciencias Agropecuarias (DACA) & 55 & 28 \\
División Académica de Ciencias de la Salud (DACS) & 56 & 28 \\
División Académica de Ciencias Económico-Administrativas (DACEA) & 38 & 19 \\
División Académica de Ciencias Sociales y Humanidades (DACSYH) & 36 & 18 \\
División Académica de Educación y Artes (DAEA) & 53 & 27 \\
División Académica de Ingeniería y Arquitectura (DAIA) & 53 & 27 \\
División Académica de Informática y Sistemas (DAIS) & 61 & 31 \\
División Académica de Ciencias Biológicas (DACBiol) & 53 & 27 \\
División Académica de Ciencias Básicas (DACB) & 57 & 29 \\
\hline Total & 462 & 234 \\
\hline
\end{tabular}

En relación con la muestra del área de conocimiento de Ciencias Sociales y Administrativas se reporta que $33 \%$ son solteros y $67 \%$ casados; $44 \%$ hombres, $56 \%$ mujeres; $10.9 \%$ pertenecen al Sistema Nacional de Investigadores (SNI); $79.7 \%$ están acreditados con el perfil PROMEP, es decir realizan en equilibrio actividades docentes, de investigación, de tutoría y de gestión administrativa (cabe mencionar que a partir del 29 de diciembre de 2013 el PROMEP recibe el nombre de PRODEP). Además, 6.3\% desempeña cargos administrativos.

El instrumento de investigación es una traducción y adaptación del cuestionario de Rizzo et al. (1970) considerando el contexto de trabajo de los docentes universitarios afiliados a cuerpos académicos; que se fue transformando a lo largo de dos proyectos de investigación, MagañA, Sánchez y Rosas (2009) y Magaña et al. (2009), y del trabajo de Surdez (2013). El cuestionario cuenta con dos secciones, la primera parte incluye variables sociodemográficas, dos con relación a la organización: antigüedad y división académica en la que labora; tres con respecto al individuo: género, edad y estado civil; y tres sobre acreditaciones y cargos administrativos: acreditar el perfil PROMEP (Actualmente PRODEP), pertenecer al SNI y desempeñar cargos administrativos. La segunda parte mide la variable ambigüedad de rol a través de cuatro dimensiones (ver tabla II), medidas cada una de ellas con cuatro reactivos en una escala tipo Likert de 5 puntos, que van desde Totalmente en desacuerdo (1) hasta Totalmente de acuerdo (5); por el sentido positivo de las frases de los reactivos, las puntuaciones más bajas denotan el problema de ambigüedad de rol.

Tabla II. Especificaciones de la variable ambigüedad de rol

\begin{tabular}{ll}
\hline Dimensión & Definición Operacional \\
\hline Autonomía & Ambigüedad en los niveles de autonomía en el desempeño del rol. \\
Demandas & Ambigüedad en las demandas de trabajo asociadas al rol. \\
Contribución & $\begin{array}{l}\text { Ambigüedad en la contribución del trabajo desempeñado por el rol } \\
\text { a los logros de la institución. }\end{array}$ \\
Normas & Ambigüedad en los objetivos y normas institucionales asociadas al rol. \\
\hline
\end{tabular}

Se evaluó la confiabilidad del instrumento a través del Coeficiente de alfa de Cronbach que reporta un valor de .926; y la validez con el análisis factorial exploratorio con rotación Varimax, el cual presenta cargas factoriales por arriba de .60 (ver tabla III), valores que indican que los reactivos están altamente correlacionados y sustentan teóricamente el constructo de cada dimensión (Hair et al., 1999; Kline, 1994 y Spector, 1992, como se cita en Morales 2011). 
Tabla III. Análisis factorial con rotación Varimax

\begin{tabular}{|c|c|c|c|c|}
\hline Reactivos & Autonomía & Demandas & Contribución & Normas \\
\hline Sé cuanta autonomía tengo en mi trabajo. & .765 & .068 & .209 & .340 \\
\hline $\begin{array}{l}\text { Puedo tomar decisiones relativas a mi trabajo sin } \\
\text { necesidad de consultar a mis superiores. }\end{array}$ & .714 & .295 & .129 & -.075 \\
\hline Conozco las decisiones que puedo tomar en mi trabajo. & .805 & .153 & .125 & .215 \\
\hline Sé exactamente lo que la institución espera de mí. & .613 & .396 & .273 & .216 \\
\hline $\begin{array}{l}\text { Recibo instrucciones clara y precisas sobre los trabajos } \\
\text { que me comisionan. }\end{array}$ & .313 & .733 & .189 & .155 \\
\hline $\begin{array}{l}\text { Son claros los métodos definidos para realizar los } \\
\text { trabajos que me demandan. }\end{array}$ & .235 & .799 & 183 & .188 \\
\hline $\begin{array}{l}\text { Son claros los indicadores de evaluación del trabajo } \\
\text { que me demandan. }\end{array}$ & .137 & .679 & .140 & .413 \\
\hline $\begin{array}{l}\text { Se definen con claridad los resultados que esperan } \\
\text { de mi trabajo. }\end{array}$ & .227 & .729 & .267 & .302 \\
\hline $\begin{array}{l}\text { Conozco cómo mi trabajo contribuye a lograr los } \\
\text { objetivos institucionales. }\end{array}$ & .186 & .313 & .846 & .151 \\
\hline $\begin{array}{l}\text { Conozco cómo mi trabajo contribuye a alcanzar las } \\
\text { metas institucionales. }\end{array}$ & .211 & .258 & .867 & .159 \\
\hline $\begin{array}{l}\text { Conozco los indicadores institucionales de calidad } \\
\text { que debo alcanzar. }\end{array}$ & .208 & .037 & .649 & .548 \\
\hline $\begin{array}{l}\text { Conozco el impacto de mi desempeño en los } \\
\text { indicadores de calidad de la institución. }\end{array}$ & .192 & .173 & .648 & .488 \\
\hline $\begin{array}{l}\text { Los objetivos de mi trabajo están claramente } \\
\text { establecidos por la institución. }\end{array}$ & .187 & .369 & .275 & .671 \\
\hline $\begin{array}{l}\text { Conozco claramente cómo los planes institucionales } \\
\text { repercuten en mi trabajo. }\end{array}$ & .231 & .164 & .228 & .685 \\
\hline $\begin{array}{l}\text { Conozco oportunamente las normas que rigen las } \\
\text { evaluaciones sobre mi desempeño. }\end{array}$ & .015 & .492 & .156 & .561 \\
\hline $\begin{array}{l}\text { La normatividad de la institución es clara con respecto } \\
\text { a mis funciones. }\end{array}$ & .150 & .397 & .161 & .678 \\
\hline
\end{tabular}

Para el análisis de datos se utilizó estadística descriptiva con el fin de identificar evidencias de ambigüedad de rol. Asimismo, para determinar la existencia de diferencias significativas entre las dimensiones de las variables de ambigüedad de rol con relación a las variables sociodemográficas se empleó el análisis de varianza ANOva y la Prueba t. Por último, con el propósito de conocer la relación de las dimensiones de ambigüedad de rol con la edad y la antigüedad se utilizó estadística inferencial a través del coeficiente de correlación de Pearson, este último debido a que estudios anteriores sobre grupos de investigación (Rosas, Magaña y Guzmán, 2008) han demostrado que a profesores con la edad y antigüedad cercana a la jubilación, pudiera implicarles un esfuerzo poco rentable capacitarse para las nuevas actividades que demandan los indicadores de productividad. Esta circunstancia podría agudizar la ambigüedad de rol en este colectivo.

\section{Resultados}

El 28\% de la muestra presenta un nivel alto de ambigüedad de rol, el 25\% un nivel moderado, el 22\% un nivel bajo y un $25 \%$ no registra ambigüedad; por tanto, el $75 \%$ de la muestra percibe cierta ambigüedad de rol en las dimensiones bajo estudio, como se observa en la tabla IV.

Tabla IV. Niveles de ambigüedad de rol, rango de valores (escala 16-80).

\begin{tabular}{lccc}
\hline Nivel de Ambigüedad & Percentil & Rango & $\%$ \\
\hline Alto & 25 & Valores $\leq 49$ & 28.1 \\
Moderado & 50 & $50-59$ & 25.0 \\
Bajo & 75 & $60-69$ & 21.9 \\
Sin ambigüedad & 100 & Valores $\geq 70$ & 25.0 \\
\hline
\end{tabular}


Los tipos de ambigüedad de rol se identificaron a través de análisis estadístico descriptivo, con el que se determinó en qué dimensiones se presentó una mayor ambigüedad de rol, resultados que se observan en la tabla V.

Tabla V. Análisis descriptivo de las cuatro dimensiones que conforman la variable "Ambigüedad de rol"

\begin{tabular}{lccccc}
\hline Dimensión & N & Mín. & Máx. & M & $\begin{array}{c}\text { Desv. } \\
\text { típ. }\end{array}$ \\
\hline Ambigüedad con la autonomía & 64 & 4 & 20 & 15.83 & 3.811 \\
Ambigüedad con las demandas & 64 & 4 & 20 & 12.55 & 4.031 \\
Ambigüedad con la contribución & 64 & 4 & 20 & 16.33 & 3.704 \\
Ambigüedad con las normas & 64 & 6 & 20 & 14.00 & 3.875 \\
N válido (según lista) & 64 & & & & \\
\hline
\end{tabular}

La mayor ambigüedad se reporta en las dimensiones Ambigüedad en las demandas y Ambigüedad en las normas, en el caso de la primera la principal causa es la falta de claridad en los indicadores de evaluación del trabajo que le demandan (como se observa en la tabla VI); en el caso de la segunda el origen más evidente es que no se conozca oportunamente los lineamientos que rigen las evaluaciones sobre el desempeño (como se observa en la tabla VII).

Tabla VI. Análisis descriptivo de la dimensión "Ambigüedad con las demandas"

\begin{tabular}{lccccc}
\hline Reactivo & N & Mín. & Máx. & M & $\begin{array}{c}\text { Desv. } \\
\text { típ. }\end{array}$ \\
\hline $\begin{array}{l}\text { Recibo instrucciones claras y precisas sobre } \\
\text { los trabajos que me comisionan }\end{array}$ & 64 & 1 & 5 & 3.38 & 1.175 \\
$\begin{array}{l}\text { Son claros los métodos definidos para realizar } \\
\text { los trabajos que me demandan }\end{array}$ & 63 & 1 & 5 & 3.22 & 1.114 \\
$\begin{array}{l}\text { Son claros los indicadores de evaluación del } \\
\text { trabajo que me demandan }\end{array}$ & 64 & 1 & 5 & 3.03 & 1.297 \\
$\begin{array}{l}\text { Se definen con claridad los resultados que } \\
\text { esperan de mi trabajo }\end{array}$ & 61 & 1 & 5 & 3.11 & 1.253 \\
\hline
\end{tabular}

Tabla VII. Análisis descriptivo de la dimensión "Ambigüedad con las normas"

\begin{tabular}{lccccc}
\hline Reactivo & N & Mín. & Máx. & M & $\begin{array}{c}\text { Desv. } \\
\text { típ. }\end{array}$ \\
\hline $\begin{array}{l}\text { Los objetivos de mi trabajo están } \\
\text { claramente establecidos por la institución }\end{array}$ & 64 & 1 & 5 & 3.72 & 1.105 \\
$\begin{array}{l}\text { Conozco claramente como los planes } \\
\text { institucionales repercuten en mi trabajo }\end{array}$ & 64 & 1 & 5 & 3.72 & 1.240 \\
$\begin{array}{l}\text { Conozco oportunamente las normas que } \\
\text { rigen las evaluaciones sobre mi desempeño }\end{array}$ & 63 & 1 & 5 & 3.10 & 1.434 \\
$\begin{array}{l}\text { La normatividad de la institución es clara } \\
\text { con respecto a mis funciones }\end{array}$ & 63 & 1 & 5 & 3.57 & 1.146 \\
\hline
\end{tabular}

El análisis ANOVA y la prueba $t$ no reportaron diferencias significativas entre las dimensiones de la variable Ambigüedad de rol (Autonomía, Demandas, Contribución y Normas) con relación a las variables sociodemográficas (Antigüedad, División Académica en la que labora, Género, Edad, Estado Civil, Perfil PROMEP, pertenecer al SNI).

Con respecto al análisis de correlación de las dimensiones de la variable Ambigüedad de rol con la edad y la antigüedad de los sujetos bajo estudio se reportaron correlaciones muy débiles, por debajo de .30, que son las que se consideran útiles (Hernández, Fernández y Baptista, 2006). 


\section{Discusión}

La investigación permitió determinar evidencias de ambigüedad de rol en la muestra de profesores investigadores del área de conocimiento de Ciencias Sociales y Administrativas de una universidad pública estatal, los tipos de ambigüedad que predominan se reportan en las dimensiones Ambigüedad con las demandas y Ambigüedad con las normas, y son menos evidentes en las dimensiones Ambigüedad con la autonomía y en la contribución del trabajo desempeñado por el rol a los logros de la institución.

Tocante a la ambigüedad en las demandas, los resultados reportan que la percepción de los profesores es que los indicadores de los trabajos que le demandan y los resultados que se esperan de su trabajo académico no son claros, esta situación se explica porque a partir de la década de los noventa uno de los elementos centrales que se evalúa es el desempeño del profesor universitario a través de diversos sistemas y programas, y con base a los resultados se asignan incentivos económicos que complementan su salario (Magaña, Surdez y Zetina, 2012), este resultado amerita una línea de investigación para precisar, de acuerdo al criterio del profesor, cuáles programas de evaluación, internos y externos a la institución, requieren de mayor claridad en lo que demandan y mejores sistemas de retroalimentación.

En este sentido, Peiró, Meliá, Torres y Zácares (1987, como se cita en Meliá et al., 1987) en el cuestionario que utilizan para identificar ambigüedad de rol (A-V) consideran como un factor la Ambigüedad de las Expectativas y Valoraciones del Conjunto de Rol, que hace referencia a la claridad de las expectativas de los superiores, compañeros e inferiores a la persona focal y la frecuencia con que ellos le dan a conocer su valoración en el desempeño del trabajo; y reportan que todos los factores de la ambigüedad de rol están relacionados significativa y negativamente con la satisfacción laboral. Asimismo, Jackson y Schuler (1985) consideran como una causa de ambigüedad la insuficiente retroalimentación de otros sobre el trabajo.

Por tanto, es importante que al profesor se le informe sobre los procedimientos a través de los cuales se les asigna determinada calificación por su desempeño y la situación por la que algunos productos académicos se le aceptan y otros no; es decir, que se sistematice una retroalimentación sobre la evaluación. Tal es el caso de la valoración de su trabajo académico a través de la percepción de los alumnos, que es muy común en las instituciones de educación superior públicas y la referente a evaluación obtenida por su productividad académica, en el marco de los programas de estímulo al desempeño del personal docente.

Concerniente a la dimensión Ambigüedad con las normas, en la muestra estudiada se origina principalmente porque no se conocen oportunamente las normas que rigen las evaluaciones sobre el desempeño, en el caso de la institución investigada esta situación es observable en el contexto del Programa de Estímulos al Desempeño del Personal Docente, que evalúa las actividades de docencia, investigación, tutorías y gestión académica, por el que se emite anualmente un reglamento con los lineamientos de operación; sin embargo, el mismo no se da a conocer a los profesores cuando inicia el período a evaluar, generando incertidumbre en el profesor en cuanto a las actividades anuales que debe desarrollar para mejorar sus indicadores y los de la institución. Este resultado coincide con causas de ambigüedad de rol expuestas por diversos autores, como son falta de claridad de objetivos de trabajo individual o alcance de las responsabilidades, e inadecuada comunicación (Slipak, 1996; Malone, 2002Por su parte, Jackson (1983, como se cita en Jackson y Schuler, 1985) concluye que la disponibilidad de información acerca del rol puede disminuir la ambigüedad.

La investigación permite concluir que en la muestra estudiada los factores asociados a la ambigüedad de rol son de contexto organizacional, no así las características individuales como la edad y la antigüedad, este resultado coincide con una de las conclusiones del meta-análisis sobre investigaciones de ambigüedad de rol de Jackson y Schuler (1985) que reporta correlaciones débiles entre la edad y la ambigüedad de rol. En cuanto a los resultados, son válidos para la población bajo estudio, por lo que su difusión puede propiciar indicativos para reorientar los lineamientos y la operatividad de los programas de evaluación del quehacer de la población estudiada por parte de la organización en la que se desempeñan; asimismo, la metodología se puede replicar en otras instituciones de educación superior, lo que permitirá hacer estudios comparativos que dén luz sobre propuestas de cambio en las políticas y 
procedimientos nacionales de evaluación del quehacer del profesor universitario.

\section{Referencias}

Aquino, S. (2005). Reflexiones en torno a la evaluación de profesores en la Universidad Juárez Autónoma de Tabasco. Perspectivas Docentes, 31, 52-56

Consejo Nacional de Ciencia y Tecnología (2014). Sistema Nacional de Investigadores. Recuperado de http://www.conacyt.qob.mx/index.php/el-conacyt/sistema-nacional-de-investigadores

De Arquer, M. I., Daza, F. M. y Nogareda, C. (1995). Ambigüedad y conflicto de rol. Notas Técnicas de Prevención edición electrónica (NTP-e 388). España: Instituto Nacional de Seguridad e Higiene en el Trabajo (INSHT). Recuperado de

http://www.insht.es/InshtWeb/Contenidos/Documentacion/FichasTecnicas/NTP/Ficheros/301a400/ntp 3 $\underline{\text { 88.pdf }}$

Edmonson, S. L. y Thompson, D. P. (abril, 2001). The "role" of burnout amog special educators: the relationship between burnout and role tension. Trabajo presentado en el Annual Meeting of the American Educational Research Association, Seattle, wA. Recuperado de

http://files.eric.ed.gov/fulltext/ED454655.pdf

Gismero-González, M. E, Bermejo, L., Prieto, M., Cagigal, V., García, A. y Hernández, V. (2012). Estrategias de afrontamiento cognitivo, auto-eficacia y variables laborales. Orientaciones para prevenir el estrés docente. Acción Pedagógica, 9(12), 87-96.

Goldman, R. y Chang, D. (Abril, 1992). The Consequence of rol conflict and role ambiguity amog junior high school administrators in Taiwan. Trabajo presentado en el Annual Meeting of American Educational Research Association, San Francisco, CA. Recuperado de http://files.eric.ed.gov/fulltext/ED346567.pdf

Gomley, D. K. (2005) Organizational climate, role ambiguity, role conflict and nurse faculty work role balance: Influence on organizational commitment and turnover intention (Tesis doctoral). Universidad de Cincinnati, $\mathrm{OH}$.

Hernández, R., Fernández, C. y Baptista, P. (2006). Metodología de la Investigación (4a. ed.). México: McGraw- Hill/Interamericana.

Ibarra, E. (1999). Evaluación, productividad y conocimiento: barreras institucionales al desarrollo académico. Sociológica, 14(41), Recuperado de http://www.revistasociologica.com.mx/pdf/4103.pdf

Izquierdo, J., Sandoval, M. C., Eslava, H. y De la Cruz, V. (2013). Sistemas de evaluación y productividad investigativa: estudio de caso con PTCS de la licenciatura de idiomas. En S. P. Aquino, D. E. Magaña y P. Sánchez (Coords.), Cuerpos Académicos en Educación Superior: Retos para el Desarrollo Institucional (pp. 209-235). México: Universidad Juárez Autónoma de México.

Jackson, S. E. y Schuler, R. S. (1985).A meta-analysis and conceptual critique of research on role ambiguity and role conflict in work setting. Organizational Behavior and Human Decision Processes, 36(1), 16-78.

León-Rubio, J. M., Cantero, F. J. y León-Pérez J. M. (2011). Diferencias del rol desempeñado por la autoeficiencia en el burnout percibido por el personal universitario en función de las condiciones de trabajo. Anales de Psicología, 27(2), 518-526. Recuperado de http://digitum.um.es/ispui/handle/10201/26557

Lloret, S., González V. y Peiró J. M. (1995). El estrés de rol en enfermeras un modelo causal. Revista de Psicología General y Aplicada, 48(3), 393-405. Recuperado de 
http://dialnet.unirioja.es/servlet/articulo?codigo $=2161481$

Magaña, D. E., Rosas, J. A, Lamoyi, C. L., Aguilar, N., Surdez, E. G., Sandoval, M. C. y Guzmán, C. (2009). Factores organizacionales relacionados al síndrome de desgaste emocional en los cuerpos académicos de la Universidad Juárez Autónoma de Tabasco (Primer Informe de investigación-CONACYT-SECTORIAL 00000000080973). Villahermosa Tabasco.

Magaña, D. E., Sánchez, P. y Rosas, J. A. (2009). Síndrome de desgaste emocional y su relación con el conflicto y ambigüedad de rol en el profesor investigador de la Universidad Juárez Autónoma de Tabasco (Informe de investigación final-PROMEP 20080783). Villahermosa, Tabasco: Autor

Magaña, D. E., Surdez, E. G., Zetina, C. D. (2012). Ambigüedad de rol en investigadores mexicanos. Revista Internacional Administración y Finanzas, 5(3), 69-87.

Malone, R. J. (2002). Tenure-track faculty socialization: the presence and effects of role ambiguity role, role conflict and role overload (Tesis Doctoral). Recuperada de la base de datos ProQuest

Meliá, J. L., Zornoza, A., Sanz, M. J., Morte, M. P. y González, V. (1987). La incidencia de los factores del conflicto de rol y de la ambigüedad de rol sobre los factores de la satisfacción laboral. Actas del Segundo Congreso Nacional de Evaluación Psicológica.

Recuperadodehttp://www.uv.es/meliaj//Papers/1987 Melia Factores.pdf

Morales, P. (2011). El análisis factorial en la construcción e interpretación de tests, escalas y cuestionarios. Recuperado de http://www.upcomillas.es/personal/peter/investigacion/AnalisisFactorial.pdf

Rizzo, J., House, R. y Lirtzman, S. (1970). Role conflict and ambiguity in complex organizations. Administrative Science Quarterly, 15(2) 150-164.

Robbins, S. P. y Coulter M. (1999).Administración (6a. ed.). México: Prentice Hall Hispanoamericana.

Rosas, J. A., Magaña, D. E. y Guzmán, C. (2008). Posibilidades de consolidación y crecimiento de los cuerpos académicos de la División Académica de Ciencias Económico-Administrativas de la Universidad Juárez Autónoma de Tabasco. Hitos de Ciencias Económico Administrativas, 14,(39) 65 - 73.

Sebastián, J. (2003). Estrategias de cooperación universitaria para la formación de investigadores en Iberoamérica. Madrid: OEl.

Secretaría de Educación Pública (2013, 29 de diciembre). Acuerdo número 712 por el que se emiten las reglas de operación del Programa para el Desarrollo Profesional. Recuperado de http://dsa.sep.gob.mx/pdfs/Reglas\%20de\%200peracion\%202014.pdf

Schulz, J. (2013). The impact of role conflict, role ambiguity and organizational climate on the job satisfaction of academic staff in research-intensive universities in the UK. Higher Education Research \& Development, 32(3), 464-478.

Schulz, J. y Auld, C. (2006).Perceptions of role ambiguity by chairpersons and executive directors in Queensland sporting organizations. Sport Management Review, 9(2), 183-201.

Slipak, O. E. (1996). Estrés Laboral. Alcmeon, 19(4). Recuperado de http://www.alcmeon.com.ar/5/19/a19 03.htm

Soto, E. (1997). La productividad ¿Nuevo paradigma del salario universitario? El caso de la UAM. Política y Cultura, 9, 149-175. Recuperado dehttp://www.redalyc.org/articulo.oa?id=26700909 
Surdez, E. G. (2013).Conflicto y ambigüedad de rol en profesores investigadores de cuerpos académicos: el caso de la Universidad Juárez Autónoma de Tabasco (Tesis Doctoral). Universidad del Mayab, Mérida Yucatán, México.

Troyano, Y., García, A. J. y Marín, M. (2006). ¿Cómo afronta el profesorado universitario la docencia en el contexto de la convergencia europea? Hacia un nuevo perfil docente. Revista de Enseñanza Universitaria, 28, 77-83. Recuperado de http://institucional.us.es/revistas/universitaria/28/06.pdf

Universidad Juárez Autónoma de Tabasco (2014). Reglamento del Programa de Estímulos al Desempeño del Personal Docente. Tabasco, México: Autor.

Zepeda, S. y Kruskamp, B. (2007), High school department chairs perspectives on instructionals supervision. The High School Journal, 90(2), 44-54. 